\title{
INDUSTRIA GLOBAL, CULTURAS Y POLÍTICAS LOCALES: LA INTERNACIONALIZACIÓN DE LA TELEVISIÓN LATINOAMERICANA
}

\author{
Silvio Waisbord*
}

Fecha de recepción: noviembre 1999

Fecha de aceptación y versión final: abril 2000

\begin{abstract}
Resumen: El artículo analiza los procesos mas importantes que experimentó la televisión latinoamericana en los ochenta y los noventa. Se presenta un argumento doble: la globalización tiene consecuencias disímiles para las televisiones domésticas debido a que la situación de la industria televisiva varía notablemente de país a país. Estas diferencias se acentuaron recientemente, precisamente, porque la globalización fortalece y debilita las posibilidades de la producción local. Las perspectivas para la producción de contenidos que reflejen culturales locales y nacionales son diferentes según factores económicos. La experiencia reciente de la televisión latinoamericana sugiere que la globalización es un proceso político-económico que abre las puertas al flujo de capitales y tecnologías y facilita la concentración de medios. Esto no implica, sin embargo, que lleve a la homogeneización de la cultura televisiva o haga irrelevante la política y el Estado como prácticas e instituciones que moldean y definen las estructuras comunicacionales de una sociedad.
\end{abstract}

Palabras clave: Televisión, globalización, producción audiovisual, medios y cultura, nuevas tecnologías.

Abstract: The article analyzes the most important processes that the Latin American television experienced in the 1980 s and 1990s. It offers a double argument: globalization has different consequences for domestic television due to the fact that the conditions of local television vary from country to country. These differences became sharper recently, precisely because globalization both strengthens and weakens the chances for local production. The prospects for the production of content that reflects local and national cultures are different according to economic factors. This case suggests that globalization is an economic-political process that opens the door to the flow of capital and technology, thereby facilitating media concentration. This does not imply, however, that it leads to the homogeneization of television cultures or that it makes politics and the State irrelevant as practices and institutions that shape and define communication structures in a society.

Keywords: Television, globalization, audiovisual production, media and culture, new technologies.

\section{INTRODUCCIÓN}

La televisión no es un bien escaso o una industria marginal en América Latina contemporánea. Su centralidad en la vida política y cultural, la existencia de poderosos grupos industriales en el sector televisivo, el crecimiento de la producción regional, y la presencia global de producciones regionales son algunas indicaciones de profundos cambios recientes. El cuadro actual de la televisión sugiere un evidente contraste comparado con los primeros tiempos en los que había un limitado número de receptores, limitadas horas de transmisión, y limitada producción local. Al mismo tiempo que las economías de la región estaban sumidas en una prolongada crisis y fueron vulnerables a recurrentes cimbronazos financieros, la industria televisiva experimentó un crecimiento notable durante las décadas de los ochenta y noventa.
De 35 millones de aparatos de televisión en 1980 se pasó a 99 millones en 1996. Traducido en porcentaje por 1000 habitantes, la cifra de 97 aparatos en 1980 subió a 204 en 1996 (UNESCO Statistical Yearbook 1998). El número de canales creció de 400 a 1500. Las cifras de televisión paga se incrementaron notablemente: segun cálculos recientes, hay 15 millones de suscriptores a televisión por cable, 2 millones a servicios de televisión por satélite y 35 millones de videocassetteras. Con variaciones por país, la television capta entre el 45 y el 60 por ciento de las inversiones anuales en publicidad. Alrededor de 170 cadenas de cable y servicios pagos son ofrecidos en la región.

* Doctorado en Sociología en la Universidad de California, San Diego. Profesor del Departamento de Periodismo y Estudios de Medios, Rutgers University, Nueva Jersey, Estados Unidos.

$凶$ waisbord@scils.rutgers.edu 


\section{ETAPAS EN EL DESARROLLO DE LA TELEVISIÓN}

No cabe duda que la televisión regional está en una nueva fase de su complicado y convulsionado desarrollo histórico. En su primera etapa durante las década de los cincuenta y los sesenta, la emergente industria tenía una cantidad reducida de receptores y horas. En la mayoría de los países, la televisión se constituyó principalmente como una industria privada con una débil presencia de la televisión pública. La estructura privada que existía en la radio se acomodó rápidamente para expandirse a la nueva industria frente a la debilidad o, en algunos países, la ausencia de intentos opuestos. La excepción a este modelo fueron dos opciones: el férreo control gubernamental durante periodos autoritarios y los proyectos que colocaron a la televisión en manos de las universidades y/o el Estado (Fox, 1997). La primera opción no cuestionó el funcionamiento privatista de la televisión sino que principalmente tuvo el objetivo de garantizar parcelas de poder e influencia para los militares de turno; la segunda, si bien limitó la participación de compañías privadas, tuvo resultados mixtos y fracasó en el intento de establecer una televisión basada sobre principios públicos. Tanto en uno como otro caso, las ambiciones privadas de organizar la televisión según principios comerciales prevalecieron 1 .

En los orígenes de la televisión, los capitanes de la industria de medios de entonces (radio y prensa) vieron en la nueva tecnología una extensión lógica de sus negocios y buscaron intensamente obtener réditos del gobierno de turno en el otorgamiento de licencias y así quedar favorablemente posicionados en el despegue de la nueva industria. Las cadenas estadounidenses surgieron en la mayoría de los casos como socios naturales de los mogules locales. Interesadas en las posibilidades del emergente mercado latinoamericano una vez que el mercado norteamericano llegó a un punto de saturación a fines de la década de los cincuenta, las cadenas tuvieron un rol protagónico como proveedores de tecnología, capital, asistencia y programación para proyectos locales.

A principios de los setenta, la televisión entró en una nueva etapa. Sacudida por los terremotos políticos que caracterizaron a la región y en el medio de debates sobre la soberanía cultural, la televisión fue tironeada entre proyectos privatistas y estatistas. Si bien algunos proyectos estatistas triunfaron con la nacionalización de canales en algunos países, no pusieron en cuestión la idea de que la televisión debe regirse por principios de mercado y mucho menos, ofrecieron una alternativa basada en la concepción de interés público. Los más importantes hitos de este período fueron la expansión sostenida del número de receptores, la distribución de señales via satélite y por cable coaxil que hizo posible la nacionalización de la televisión, y la introducción de transmisiones en color.

Uno de los rasgos más importantes de este período fue la consolidación de la producción local y la reducción de la programación importada (sobre todo en horario central) en algunos países. Mientras que este proceso fue visible en Brasil, México y Venezuela, en otros países la producción continuó siendo mínima y los canales ofrecían mayormente programación extranjera. A partir de este momento las diferencias entre distintos mercados televisivos se profundizaron. La television en la región se transformó en un complicado mosaico de desarrollos, presentando situaciones que a pesar de tener rasgos similares, son bastantes heterogéneas. Estas diferencias están dadas por distintos factores que, como se explica más adelante, están mutuamente vinculados: el tamaño del parque televisivo, el desarrollo de la industria audiovisual en general, la cantidad de inversión publicitaria y producto bruto nacional, y las desigualdades en el desarrollo de la televisión paga.

Propongo entender estas diferencias como la piedra Rosetta del análisis de la televisión latinoamericana en su tercera y presente etapa. La aparente unidad que la televisión regional tuvo, de acuerdo a estudios que hablaban de la televisión latinoamericana en singular; fue resquebrajada en los setenta y los ochenta. Tales diferencias se acentuaron en los noventa, conformando un panorama que varía notablemente de país en país. Mi interés en este artículo es analizar los procesos más importantes de la década de los noventa y desarrollar un argumento doble: al acentuarse las diferencias en la industria televisiva, la globalización tiene consecuencias disímiles para las televisiones domésticas que escapan a las generalizaciones fáciles. Esto ofrece diferentes perspectivas para la producción de contenidos que reflejen culturas locales y nacionales. La experiencia reciente de la televisión latinoamericana sugiere que la globalización es un proceso político-económico que abre las puertas al flujo de capitales y tecnologías y facilita la concentración de medios. Esto no implica que la globalización conduzca a la homogeneización de la cultura televisiva o haga irrelevante la política y el Estado como prácticas e instituciones que moldean y definen las estructuras comunicacionales de una sociedad.

\section{NUEVOS MEDIOS Y NUEVAS POLÍTICAS}

A fines de los ochenta, sólo unos pocos observadores podían imaginar que la televisión en la región experimentaría sustanciales transformaciones. Estas transformaciones fueron tanto legales como tecnológicas, políticas, económicas y financieras. Globalización, el proceso que profundamente cambiara las estructuras y dinámicas de las industrias de medios en el mundo, también afectó a América Latina. Las transformaciones en la región estuvieron en sintonía con la implementación de políticas de libre mercado en otras regiones. La trinidad de privatización, liberalización y desregulación fue adoptada en las políticas de televisión (y de medios en general). Aunque si bien los sistema televisivos ya estaban regidos por principios comerciales, la globalización definió el tironeo histórico entre estado y mercado en favor de este último, eclipsando las posibilidades de cualquier alternativa.

1. Es importante indicar, sin embargo que actualmente la situación de la televisión pública no es idéntica en la región. En países como Chile y Colombia, o en el caso de TV Cultura en São Paulo, la idea de servicio público es influyente en la organización y la programación de canales mantenidos con fondos públicos, a diferencia de otros países donde claramente la noción de canales públicos como propiedad estatal/gubernamental se perpetúa. 
En los noventa, la adopción de políticas favorables a intereses privados pusieron un aparente punto final a la larga batalla entre modelos estatistas y privatistas. Ciertamente, tales políticas sintonizaron con la implementación general de políticas de libre mercado en la economía en general. En Argentina y México, la privatización de canales estatales que habían sido nacionalizados a principios de los setenta, catalizó cambios sustanciales. En Bolivia y en Chile, se autorizó al capital privado la propiedad de canales de televisión que hasta entonces había quedado en manos de universidades. La propiedad privada de los canales fue también aprobada en Colombia donde el estado había controlado la televisión y limitado al sector privado a la producción de programas con topes fijos. Firmas privadas también se vieron favorecidas por la transición de una era de escasez a la era de abundancia del espectro electromagnético ya que recibieron la gran mayoría de las nuevas frecuencias adjudicadas.

Junto con la privatización, la eliminación de restriciones en la propiedad multimedios y la liberalización de distintas áreas permitieron un proceso de rápida concentración y consolidación de empresas televisivas. La existencia de grandes grupos en la región no es nueva. Antes de los noventa ya existían grupos como Globo, Televisa y otros conglomerados que pugnaban por estar integrados vertical y horizontalmente. Un número de decisiones legales aceleró este proceso, decisiones que en varios casos estuvieron en contradicción con reglas constitucionales que prohiben la formación de monopolios y, específicamente, en las industrias de medios. Tales políticas abrieron viejos y nuevos sectores y fortalecieron la posición de fuertes grupos locales. Grupos como Clarín en Argentina y RCN y Caracol en Colombia, por ejemplo, fueron beneficiarios de la privatización, lo cual les permitió controlar recursos fundamentales en la industria televisiva, una industria clave para las estrategias empresariales de solidificar su posición como conglomerados multimedios. La privatización también permitió que distintos intereses industriales se expandieran rápida y agresivamente en la televisión, como el Grupo Elektra en Mexico que formó Azteca TV después de recibir las licencias por los canales 7 y 13. Para cualquier empresa con ambiciones locales, regionales y globales, una sólida posición en la industria de la televisión abierta es fundamental ya que esta recibe la parte del león de las inversiones publicitarias y es el principal canal para la producción y exportación de programas.

\section{LA TELEVISION PAGA}

La evolución de la televisión paga y en particular la apertura del mercado de cable también introdujeron nuevas dinámicas. Durante años, analistas financieros y de la industria vienen augurando un enorme futuro para la televisión paga en la región ya que sólo un 15\% del total de hogares con televisión reciben servicio de cable o satélite (Connor, 1997; Kilby, 1997).

La industria del cable se desarrolló en tiempos diferentes $\mathrm{y}$ en condiciones particulares en distintos países. El boom se produjo claramente en los noventa en la mayoría de los países. Como muestra el Cuadro I, la penetración del cable en números absolutos es desigual en la región.

Cuadro I: Televisión por cable en América Lat ina y el Caribe, 1998

\begin{tabular}{|l|r|}
\hline Argentina & $5,096,000$ \\
\hline México & $2,351,000$ \\
\hline Colombia & $2,235,000$ \\
\hline Brazil & $1,745,000$ \\
\hline América Central/ Caribe & $1,218,000$ \\
\hline Chile & 906,000 \\
\hline Venezuela & 536,000 \\
\hline Resto & $1,064,000$ \\
\hline Total & $15,151,000$ \\
\hline Fuente: Audits \& S urveys 1998 & \\
\hline
\end{tabular}

La proporción de la televisión paga es desigual a lo largo de la región. Cerca del $60 \%$ de los hogares son suscriptores de cable en Argentina, $25 \%$ en Chile y Mexico, $16 \%$ en Venezuela, y menos del $10 \%$ en Brazil y la mayoría de los otros países. En el marco de persistentes desigualdades sociales, la distribución también está altamente estratificada, ya que las audiencias de cable suelen concentrarse principlamente entre clases altas y medias.

La industria del cable pasó a ser mayormente parte de las compañías establecidas. Los grupos Cisneros y Televisa controlan un importante número de suscriptores en Venezuela y México respectivamente. En la mayoría de los países, el desarrollo del cable fue fragmentado en sus comienzos y gradualmente pasó a manos de unos pocos grupos, algunos ya establecidos y otros que entraron a la industria mediática con la irrupción de la televisión paga. Varios mercados de cable son duopolios como Argentina donde los grupos Clarín y CEI detentan el $80 \%$ del mercado, Brasil donde Globo y TVA tienen $70 \%$ y $30 \%$ respectivamente del total, o Ecuador en el que TV Cable y Univisa tienen la mayoría de los abonados. Otros mercados son controlados por tres empresas: los tres canales de televisión abierta se expandieron hacia la nueva industria en Uruguay, y Cabletel, InterCable y Supercable son dueñas de la mayoría de los suscriptores en Venezuela.

Asimismo, los grupos más fuertes tomaron la delantera en la televisión satelital. Los pronósticos optimistas de 5 millones de suscriptores para principios del 2000, basados en las dificultades y los costos de cablear extensas y difíciles geografías y expectativas de crecimiento económico, alimentaron los lanzamientos de dos servicios regionales (Paxman, 1999b). Uno es Galaxy Latin America en el que se encuentan asociados Hughes, Cisneros de Venezuela, Abril de Brazil, Multivision de Mexico, y Clarín de Argentina. El otro es Sky Latin America que reune a News Corporation, TCI, Televisa, y Globo. Ambos muestran que el camino para macro proyectos regionales de televisión deben reunir compañías de tecnología y multimedios globales con los productores mas importantes de televisión en América Latina. Mientras que los gigantes de las telecomunicaciones globales ponen sobre la mesa una vasta experiencia mundial, extensos archivos audiovisuales y el cachet para financiar costosos proyectos, los socios regionales ofrecen experiencia doméstica y programas aceptados por las audiencias. Hasta el momento, los resultados no han sido como los pronósticos auguraban debido a los 
altos costos iniciales de la televisión directa al hogar y otros problemas (coordinacion entre socios, falta de programas de Televisa en Sky).

\section{V. ¿QUÉ TRAJO LA GLOBALIZACIÓN?}

La privatización y la liberalización acentuaron la internacionalización de la industria de la televisión. Si bien la internacionalización en décadas pasadas era sinónimo de la presencia de las cadenas norteamericanas en la televisión latinoamericana, la variedad de flujos y contraflujos actuales sugieren que la globalización es mas compleja que en el pasado.

Uno de los desarrollos más importantes fue la expansión de grupos dominantes de mercados grandes hacia mercados medianos y pequeños. Por una parte, el ingreso fue posible a través de la compra de acciones parciales o totales en canales. Con el objeto de asegurarse "ventanas de exhibición" en mercados regionales, grupos como Televisa, Azteca, Cisneros, y Gonzalez adquirieron parcial o totalmente canales en Chile, Ecuador, El Salvador, y Perú. La programación de canales como América TV en Perú o en Ecuador, por ejemplo, en las que González y Televisa respectivamente tienen participacion directa, refleja que son claramente bocas de salida para producciones realizadas en México y en Miami. Por otra parte, las redes de cable y satélite también permitieron una presencia regional. Aunque la mayoría de las señales en cada país ofrecen programas extranjeros, la mayoría son hablados en español. Los grandes productores de programación tienen sus servicios regionales tales como Telefé de Argentina, SBT y Manchete de Brasil, Universidad Nacional de Chile, Eco/Televisa de México, IBC/RCTV de Venezuela (a través de GEMS). Además hay un número considerable de pequeñas señales con recursos limitados que tienen alcance local o nacional.

A pesar de tener reducidas audiencias, la apertura de sistemas de cable estimuló el crecimiento de la producción local en mercados medianos y grandes. A mayor demanda se abrieron las perspectivas para la producción para señales domésticas y redes regionales ${ }^{2}$. A diferencia de los sistemas de cable en Estados Unidos donde las grandes programadoras conquistaron rápidamente el espectro del cable dejando limitado o nulo lugar para productoras independientes, en América Latina, la oferta de programación fue originariamente menor que el número de canales disponibles. Esto permitió que pequeñas y medianas productoras, incluso con escasos recursos, accedieran a las nuevas señales. Si bien la gran mayoría de las señales con mayor alcance panregional proviene de Estados Unidos, el panorama es más diverso a nivel nacional. Los sistemas de cable típicamente ofrecen entre 60 a 70 canales de los cuales una tercera parte son cadenas norteamericanas, otra tercera parte son regionales y el resto son locales (incluidas las señales de canales abiertos) más un puñado de canales europeos. Estos datos sugieren que la televisión paga permite la expansión de las programadoras y estudios globales pero no asfixia la producción local y regional. El proceso originario de expansión de la oferta de canales modestos en mercados con un apreciable volumen de producción, sin embargo, amenaza con revertirse a medida que los grandes grupos locales y extranjeros se expanden en un número limitado de canales. La concentración desenfrenada excluye la posibilidad de que el cable ofrezca oportunidades para diferentes fuentes de programación y, de este modo, permite aun más la consolidación de las grandes empresas.

Asimismo, la liberalización y desregulación de la televisión por cable facilitaron la expansión de firmas globales de hardware como TCI y Liberty Media que son activas en mercados con un alto, real o potencial, número de suscriptores y de hogares con televisión. Dado el alto número de suscriptores en Argentina, comparable a los países mas cableados en el mundo, ésta se convirtió en la plataforma para proyectos regionales de televisión paga. El tratado comercial de inversiones recíprocas de 1994 entre Argentina y Estados Unidos en gran medida hizo esto posible ya que facilitó la entrada de grupos norteamericanos. En el contexto de otras decisiones que facilitaron la inversion privada y extranjera, el tratado introdujo notables cambios estructurales en la industria de medios argentina. Asimismo, en Brasil, la ley del cable de 1995 inició transformaciones en el sistema de televisión paga. Al incrementar la participación extranjera al $49 \%$, la ley señaló un giro importante frente a la tradición proteccionista que caracterizó a Brasil. A pesar de tener un número reducido de suscriptores, Brasil es regularmente citado por analistas de la industria como el "gigante dormido" de la televisión paga debido al tamaño de su población y de inversiones publicitarias.

Como ocurrió en otras regiones, varias firmas financieras globales también han entrado en la industria de medios en América Latina. Goldman Sachs, Citibank, Hicks, Muse, Tate \& Furs, y Merill Lynch entre otros manejan una cantidad apreciable de intereses mediáticos. Necesitados de capital para financiar adquisiciones, mantener el ritmo de expansión, y superar a la competencia, grupos locales acudieron a poderosas firmas financieras. Asimismo, la participación de poderosos socios financieros otorga garantías a quienes controlan acciones de grupos mediáticos ya que, preocupados por excesivos gastos ocasionados por la expansión vertical y horizontal en el contexto de economías tambaleantes, temen por la caída de los bonos de empresas mediáticas e incluso por su quiebra. Para los grupos financieros, la decisión de entrar en la industria de medios respondió a la estrategia de asegurarse una posición dominante en economías recientemente privatizadas de la región, particularmente en una industria dinámica y altamente redituable como la de medios. El lugar clave del Citibank en los medios argentinos (a través del Citibank Equity Investment CEI) se originó en la decisión de la administración Menem de privatizar un considerable número de compañías estatales a principios de los noventa. Citibank, uno de los principales acreedores de la deuda pública argentina, pudo asi

2. En su estrategia de abaratar costos y ofrecer programación regional, algunas de las grandes cadenas regionales como MTV y Discovery han comprado recientemente producciones locales (videos musicales y documentales respectivamente), programas que no sufren demasiado descuento cultural, es decir, que no están fuertemente ligados a cuestiones nacionales. 
canjear títulos de deuda pública por intereses activos. El fondo de inversión Hicks, Muse, Tate \& Furs, activo en varios mercados de la región como Brasil y Argentina, también se expandió en la industria de medios y se convirtio en un «player» central en la industria mediática argentina. Además de participar en el CEI y tener intereses en firmas de cable, el fondo controla $49 \%$ de la compañías que tienen los derechos de transmisión de fútbol en Argentina, Brasil y Uruguay $^{3}$.

\section{EL AUMENTO DE LA PRODUCCIÓN LOCAL}

Con la llegada de la televisión paga, la apertura de mercados regionales y el otorgamiento de nuevas licencias de canales, se multiplicaron las ventanas de exhibición. Esto aumentó la demanda de horas de programación. Algunas estimaciones calculan que el número anual de horas de televisión se triplicó durante los noventa (Catalán, 1999). Esta situación, en principio, hubiera favorecido solamente o principalmente a Hollywood, como temieron razonablemente observadores europeos debido a la privatización de los sistemas televisivos y el surgimiento de nuevas tecnologías para la distribución de señales durante los ochenta. No hay otra industria televisiva que pueda competir con Hollywood en términos de volumen de producción, redes de distribución internacional, familiariedad de audiencias con contenidos, y archivos de programación. Sin embargo, el aumento de demanda de contenido creada por la apertura de nuevas señales dio lugar a resultados diferentes de los anticipados.

Si bien no hay estimaciones exactas, es indudable que el número de horas de televisión a nivel local y regional aumentó considerablemente en las últimas decadas. ¿Cómo explicar este incremento? En primer lugar, el menor costo de las tecnologías de video y otros insumos eliminaron importantes obstáculos que, especialmente durante las primeros tiempos de la televisión, operaron en contra de la producción local y favorecían a los enlatados importados. Actualmente, en cambio, no es difícil incluso para canales con escasos recursos acceder a las tecnologías necesarias para la producción. Mas aún, grupos dominantes como Televisa, Globo y SBT son dueños de inmensos estudios equipados con las últimas tecnologías para producir miles de horas de programación anuales más un número de produciones cinematográficas.

Segundo, la preferencia de los canales por la programación local también incentiva la producción doméstica. Esta es atractiva por la simple razón que, consistemente, supera a producciones regionales o norteamericanas en ratings. Telenovelas, comedias, dramas, deportes, y noticieros se encuentran al tope de los rankings, confirmando que, cuando existe, la producción doméstica supera a los enlatados importados. Si tomamos datos de Diciembre de 1999, vemos que los diez programas más vistos en Argentina, Colombia y México fueron producciones locales (Blejman, 1999; IBOPE, 1999a, 1999b). En mercados mas modestos como Perú, los resultados fueron similares a excepción de un anime japonés (La República, 1999). Para una industria que se guía por los resultados de las medi- ciones de audiencia, estos datos son fundamentales en la toma de decisiones. En la mayoría de los casos, los precios de los programas importados son sensiblemente más baratos, particularmente comparados con los costos de producciones dramáticas o cómicas, pero atraen bajas audiencias $y$, por consiguiente, menores ingresos publicitarios. En un mercado mediano como Chile, el costo promedio de una hora de telenovela oscila entre 30 y $40 \mathrm{mil}$ dólares, bastante más que un enlatado; sin embargo, tiene mayores chances de tener mas rating que una serie extranjera. Considerando que el costo de medio minuto de publicidad es 4,000 dólares durante programas de alta audiencia en horario central, lo que multiplicado por 15 minutos por hora da réditos considerables, es obvio que los canales prefieren producciones locales (Capital, 1998). En mercados pequeños, en cambio, donde el costo de producciones importadas es sustancialmente más bajo, oscilando entre los 500-800 dólares por hora, y las inversiones publicitarias son menores, la producción local enfrenta dificultades insalvables. Estas diferencias se agudizan cuando no existe (o no es aplicada) legislación que proteja a la industria local y no hay apoyo oficial en forma de subvenciones, quitas de impuestos, o políticas de promoción.

En la mayoría de los países, la programación importada suele ser regional mas que norteamericana (producciones de otros países son casi inexistentes en la televisión abierta), salvo películas y dibujos animados, géneros que todavía enfrentan particulares problemas en la región debido a que demandan mayores inversiones. Salvo excepciones, los enlatados de Hollywood suelen ser usados como fillers en horarios marginales, mientras que es habitual que producciones regionales sean programadas en horario central o constituyan el núcleo de las telenovelas de la tarde. La presencia de programación regional, particularmente en horarios claves como las primeras horas de la tarde y el horario central, se explica por la «proximidad cultural» (Hoskins, McFayden and Finn, 1997) que tienen esas producciones con las audiencias locales. La distancia cultural entre audiencia y programación es menor (lenguaje, costumbres, hábitos, historias). No todas las producciones regionales, sin embargo, tienen las mismas chances de cruzar las mismas fronteras nacionales/culturales. La observación apócrifa que América Latina es una región desunida por un mismo idioma es relevante en este contexto ya que a pesar de ser en castellano, el origen de la programación notablemente determina su facilidad (o dificultad) en cruzar acentos locales y nacionales. Si bien las barreras linguísticas son menores que en regiones con idiomas diferentes, la aceptación (o rechazo) de acentos es una importante defensa cultural que facilita o dificulta la exportación de programas. La ausencia de "políticas de defensa audiovisual" no implica la ausencia de defensas

3. Aquí es importante mencionar la carrera para controlar la televisación de fútbol entre varias compañías. Esto garantiza el control de una de las fuentes más importantes y redituables de programación. En algunos casos, los derechos son controlados por empresas relativamente nuevas como Torneos y Competencias que no sólo detenta el control en Argentina (su base originaria) sino también en Uruguay y Chile. En otros casos como Televisa y Azteca, la misma empresa es parte de conglomerados industriales propietarios de los más populares equipos nacionales, lo cual les garantiza grandes audiencias y bajos costos. 
dadas por la preferencia de las audiencias de reconocerse en las pantallas o ver culturas que son familiares.

¿Cómo explicar sino que programas mexicanos dominan las pantallas en América Central, colombianos y venezolanos en Ecuador, y argentinos en Paraguay y Uruguay? En parte estos flujos se explican por razones estrictamente económicas y de mercadeo. Grupos mexicanos (Televisa, Azteca y Gonzalez), por ejemplo, tiene aseguradas bocas de salida al ser propietarios de canales en varios países centroamericanos, tuvieron la ventaja de ser first-movers como vendedores de programas en mercados en formación y pequeños, y han comercializado agresivamente sus producciones desplazando a posibles competidores. Sin embargo, factores culturales también explican la aceptación de programas de algunos (y no todos) los países. En los ejemplos mencionados, las audiencias locales están familiarizadas con la televisión de esos países y aceptan sus acentos. Programas argentinos, por ejemplo, son difíciles de exportar al norte de Ecuador ya que las audiencias no están acostumbradas e incluso rechazan el acento 4 .

Tercero, el incremento de la producción doméstica obedece a la intención de las compañías televisivas de conquistar otros mercados. Mientras que los sistemas televisivos regionales y globales mantuvieron políticas proteccionistas y estaban centrados alrededor de la televisión pública o estatal, había pocas o nulas posibilidades de que los productores regionales pudieran recuperar costos o tener ulteriores ganancias. La apertura de mercados internacionales en los noventa sumada a la positiva respuesta que han tenido las producciones latinoamericanas (particularmente telenovelas) en Europa del Este, Medio Oriente y Asia cambió el panorama al ofrecer un nuevo incentivo para la programación local. Para las compañías con tradición de vender programas al exterior (Televisa, Globo, Venevisión, RCTV), la exportabilidad de sus producciones ha sido una preocupación constante, si bien el mercado local ha sido, y sigue siendo, fundamental. En la última década se amplio el pool de exportadores con el ingreso y la expansión de las operaciones de productores de Argentina, Colombia, y Chile al escenario internacional. Si bien los mercados globales son secundarios ya que las decisiones de producción se guían principalmente por el resultado de una producción a nivel local, su existencia introdujo una variable importante que ofrece posibilidades comerciales inexistentes en décadas anteriores 5 .

Una novedad importante es el surgimiento de productoras independientes, lo que se ha llamado el proceso de «tercerización» (Paxman, 1999a). A diferencia de los casos de Globo y Televisa en los que el mismo conglomerado produce, distribuye y exhibe programas, han surgido empresas independientes que abastecen programas a canales (o co-participan en la producción y distribución posterior en otros mercados). Es el caso de Ideas del Sur, Cuatro Cabezas, Pol-Ka, y Promofilm en Argentina, Argos Producciones en México, Iguana Producciones en Perú entre otros, que produjeron algunos de los más exitosos programas en los últimos años, varios que incluso fueron exportados. Esto obedece a que los canales (Artear y Telefé en Argentina, Azteca en México), asegurados en su posición dominante en los mercados locales, optaron por no correr el riesgo de invertir en gastos de producción particularmente en años que las perspectivas de la televisión abierta fueron inciertas. Como consecuencia del tobogán económico y financiero que experimentó América Latina, muchos canales abiertos tuvieron balances en rojo en los últimos años de los noventa. Esta situación los llevó a reducir planteles de producción y delegar la tarea a terceros. Por otra parte, cualquier productor con aspiraciones de colocar programas en la televisión abierta (y expectativas de entrar a mercados internacionales), no tiene demasiadas opciones a nivel local. Es netamente un mercado de compradores ya que hay dos o a lo sumo tres canales a los que les puede vender o entrar en coproducción.

Otros factores que también impulsaron la producción local son las políticas de protección y el crecimiento del cable. Aunque las regulaciones y cuotas existentes son rara vez monitoreadas, las disposiciones que estipulan horas mínimas de contenido local o prohiben programas extranjeros en horarios centrales han resultado favorablemente en un incremento de la producción doméstica ${ }^{6}$. Además, la expansión del cable en Argentina, Chile y México hizo que los canales abiertos desistieran de pasar enlatados norteamericanos (películas, comedias y dramas) en horarios centrales ya que estos programas suelen ser ofrecidos por las cadenas norteamericanas de cable y satélite (Fox, HBO Ole, USA y otras) con el servicio básico de cable antes de otorgar los derechos para la televisación local. A medida que el cable creció, la audiencia para esas producciones disminuyó, particularmente sectores medios y medios-altos que tiene acceso a cable y son de principal interés para los avisadores.

El aumento de la producción local no implicó inversiones de gran magnitud. No es inusual que talk-shows, programas de juegos y de variedades sean comunes ya que tiene bajos costos y permiten publicidad dentro y fuera de la programación. Estos programas tienen nulas chances de

4. Entrevista con Cesar Diaz, Telefe Internacional, Diciembre de 1999. También cabe indicar que el problema no es tanto el posible descuento cultural que sufre la programación ya que, a pesar de diferencias según el país de origen, fundamentalmente se trata de telenovelas que cuentan historias básicas y conocidas por las audiencias de la región. Es bien sabido que las telenovelas brasileras son diferentes de las mexicanas $y$ de las venezolanas, pero la cuestión no es tanto el tipo sino el acento del programa. En países donde la producción doméstica es escasa y se limita a noticieros, talk-shows y deportes, programación más cara como las comedias, dramas y programas de variedades de televisoras vecinas es preferida.

5. A pesar de la creciente presencia de producciones latinoamericanas en mercados globales, sólo en casos excepcionales la programación tiene en cuenta audiencias internacionales. Las costosas producciones de Globo o telenovelas argentinas protagonizadas por las mismas estrellas de éxitos anteriores en mercados internacionales (que insumen costos que no pueden ser pagados solamente por la distribución doméstica), o casos de telenovelas que al ser pensadas como productos de exportación tienen contenidos menos locales y lenguaje mas «universalizado», son algunos ejemplos que indican la importancia de la globalización de la televisión para la producción local. Telenovelas «de época» o documentales que tienen costos por arriba del promedio, sólo pueden ser redituables si son distribuidos internacionalmente.

6. Por ejemplo, la legislación venezolana estipula que un $50 \%$ de la programación semanal deber ser nacional, pero este mínimo no es regularmente cumplido. 
ser exportables pero proveen una solución fácil y económica para completar grillas de programación. Sólo incentivados por el éxito de dramas o telenovelas en canales rivales o por las perspectivas de colocar programas en otros mercados, algunos programadores abandonan programación barata. La preferencia por géneros baratos se acentúa durante las crisis económicas cuando las inversiones publicitarias se achican y se buscan programas que no requiren actores, guionistas, filmacion en exteriores, grandes equipos y largas horas de producción. Durante tales épocas, habituales en una región sacudida por reiteradas debacles económicas y financieras, los programadores decidieron ocupar la pantalla con shows regionales que demandan menores gastos que las inversiones en programación doméstica. Esto sucedió incluso en países con fuerte producción local como Brasil donde la cadena SBT recurrió a telenovelas mexicanas para cubrir horarios y la Bandeirantes suspendió la producción de miniseries cuando la crisis del 97 y la devaluación del 99 sustancialmente bajaron el paquete publicitario. En la misma época, telenovelas mexicanas, colombianas y brasileras invadieron las pantallas venezolanas, lo cual fue sintomático considerando que las dos principales cadenas (Venevisión y RCTV) fueron pioneras en la región en la producción y exportación de programación 7

Otra alternativa es la compra de formatos. Llenar grillas de programación con programas de juegos o talk-shows basados en formatos es más barato que producir telenovelas y dramas o comprar producciones independientes. A diferencia de épocas anteriores, los dueños de formatos tiene mayor capacidad para monitorear la "utilización" no autorizada de sus ideas en otros países y vigilar el cumplimiento de contratos de programación. Esto hace que las televisoras locales recurran a la compra de formatos como alternativas baratas y probadas. No sólo han adquirido formatos estadounidenses y europeos sino tambien regionales. Telefe de Argentina vendió el formato de Chiquititas, una telenovela para niños con gran éxito local, a la SBT de Brasil (con buenos resultados) y a Azteca de México (en esta última la experiencia no funcionó debido a varios problemas). En algunos casos, para gerentes de programación la compra de formatos es preferible a los programas enlatados porque permite adquirir una fórmula probada que puede ser adaptada a rasgos locales tales como acentos, lugares y escenografías (en telenovelas) o características particulares de la audiencia y el país (en el caso de programas de juego).

\section{UN MERCADO DESIGUAL}

El incremento de la producción televisiva no se produjo en forma similar en toda la región, sino principalmente en mercados grandes y medianos. El caso latinoamericano confirma que mercados con un importante número de hogares con televisión, sustanciales inversiones publicitarias y considerable producto bruto interno ofrecen mejores condiciones para estimular la producción televisiva. Como muestra el cuadro II, hay profundas disparidades entre los mercados televisivos de la región.
Cuadro II. Tamaño y características de mercados de televisión en América Lat in a

\begin{tabular}{|c|c|c|c|c|}
\hline & $\begin{array}{l}\text { Población } \\
\text { (mil lones) }\end{array}$ & $\begin{array}{c}\text { Producto Bruto } \\
\text { (miles de millones de } \\
\text { dólares) }\end{array}$ & $\begin{array}{c}\text { Hogares } \\
\text { (millones) }\end{array}$ & $\begin{array}{c}\text { Hogares TV } \\
\text { (millones) }\end{array}$ \\
\hline Argentina & 36.9 & 294.6 & 10.6 & 9.5 \\
\hline Bolivia & 7.9 & $\begin{array}{r}24.0 \\
6.1\end{array}$ & 1.9 & 1.0 \\
\hline Brasil & 171.8 & 748.9 & 36.0 & 34.5 \\
\hline Chile & 15.0 & 74.2 & 4.1 & 3.5 \\
\hline Colombia & 39.3 & 85.2 & 7.2 & 6.5 \\
\hline Costa Rica & 3.7 & 9.0 & 0.6 & 0.5 \\
\hline Eauador & 12.5 & 19.0 & 2.1 & 0.6 \\
\hline El Salvador & 5.8 & 10.5 & 1.1 & 1.0 \\
\hline Guatemala & 12.3 & 15.9 & 1.8 & 1.1 \\
\hline Honduras & 6.0 & 4.0 & 0.9 & 0.4 \\
\hline Mexico & 100.3 & 334.8 & $\begin{array}{r}0.9 \\
19.0\end{array}$ & $\frac{0.4}{16.0}$ \\
\hline Nicaragua & 4.7 & 2.0 & 0.7 & 0.6 \\
\hline Panamá & 2.8 & 8.2 & 0.5 & 0.4 \\
\hline Paraguay & 5.4 & 9.6 & 1.2 & 0.7 \\
\hline Perú & 26.6 & 60.9 & 4.2 & 3.1 \\
\hline Uniguay & 3.3 & 18.1 & 0.9 & 0.8 \\
\hline Venezuela & 23.2 & 67.3 & 4.9 & 3.9 \\
\hline
\end{tabular}

Las «ventajas comparativas» (Hoskins and Mirus, 1988; Waterman, 1988; Wildman, 1994) de cada mercado explican las notables diferencias en la cantidad de horas producidas en distintos países de la región. Brasil con un mercado publicitario anual estimado en 3.800 millones de dólares o México con 1.500 millones ofrecen claramente mejores condiciones para la consolidación de una industria televisiva que el resto de la región. El paquete publicitario es considerablemente menor en otros mercados: 250 millones en Chile, 180 en Colombia, 30 en Panamá, 25 en Uruguay y 18 en Nicaragua (Alvarez, 1993; Athayde 1999; Capital, 1998, Paxman and Sutter, 1997; Semana, 1999). La televisión brasilera y la mexicana tienen acceso a una torta publicitaria varias veces mayor que sus pares en el resto de la región, lo cual le permite producir más horas e invertir mayores recursos. Como consecuencia, es menos probable que en los grandes mercados haya un alto número de producciones extranjeras (tanto norteamericanas como regionales). Por el contrario, los mercados menores presentan grillas televisivas con producción local barata (noticieros, talk-shows, variedades) mezclados con series, películas y dibujos animados estadounidenses y japoneses, y telenovelas y programas de variedades procedentes de Miami y países de la región (principalmente Mexico, Brasil y Venezuela)

Las diferencias en la capacidad de producción entre países también pueden notarse en el Cuadro III.

\section{Cuadro III. Preferencia de tele nove las según origen (en porcentaje)}

\begin{tabular}{|l|c|c|}
\hline Pais & Local & Regional \\
\hline Argentina & 31 & 27 \\
\hline Bolivia & 33 & 42 \\
\hline Brasil & 73 & 9 \\
\hline Chile & 49 & 31 \\
\hline Colombia & 39 & 23 \\
\hline Costa Rica & 25 & 48 \\
\hline República Dominicana & 7 & 25 \\
\hline Ecuador & 37 & 52 \\
\hline El Salvador & 6 & 39 \\
\hline Guatemala & 21 & 14 \\
\hline Honduras & 14 & 51 \\
\hline México & 32 & 12 \\
\hline Nicaragua & 4 & 24 \\
\hline Panamá & 13 & 65 \\
\hline Paraguay & 4 & 51 \\
\hline Perú & 30 & 22 \\
\hline Uruguay & 4 & 29 \\
\hline Venezuela & 46 & 41 \\
\hline Fuente Audits \& Surveys 1998 & & \\
\hline
\end{tabular}

Fuente: Audits \& Surveys 1998

7. No todo fue pesar para varios productores. La crisis en países asiáticos y en Europa del Este en 1997 aumentó la demanda de produc ciones extranjeras, incluidas telenovelas latinoamericanas ya que su costo es inferior al de producción doméstica. 
Ademas de mostrar la popularidad de las telenovelas en la región, este cuadro sugiere que en países con producción importante (Argentina, Brasil, Chile, Colombia, México, Perú y Venezuela), las telenovelas locales son preferidas sobre las regionales. En los grandes productores (Brasil y Mexico), las telenovelas extranjeras son contadas y el porcentaje de audiencia que las ve es significativamente bajo ( 9 y 12 respectivamente). En cambio, en países con baja producción (Bolivia, Costa Rica, República Dominicana, Ecuador, El Salvador, Honduras, Nicaragua, Panamá, Paraguay y Uruguay), inevitablemente la mayoría de la audiencia mira telenovelas de otros países ${ }^{8}$. Esto sugiere no sólo que explicaciones culturalistas son correctas ("las audiencias prefieren programas que sean familiares culturalmente") sino también muestra que argumentos económicos son necesarios para entender el tráfico de programas en la región.

No sólo el tamaño del mercado determina las condiciones para la producción doméstica. Su estructura también es importante ya que, en su mayoría, se trata de mercados altamente concentrados con empresas que han mantenido estrechos contactos con poderes gobernantes. Tanto Globo como Televisa no sólo han tenido acceso a las más grandes mercados publicitarios y televisivos sino que también han detentado posiciones quasi-monopólicas durante décadas en dos de los mercados geolinguísticos más grandes (Sinclair, 1999). La proximidad a gobiernos autoritarios sumada a las ventajas que tenían en sus respectivos mercados colocó a ambas compañías en una posición ventajosa para producir miles de horas anuales. Al no haber tenido prácticamente competencia en años que fueron fundamentales en la constitución de la industria televisiva, se aseguraron que su programación, mas allá de méritos propios, capturara una considerable audiencia y la mayoría de los gastos en publicidad en los mercados más grandes de la región. Esta hegemonía doméstica otorgo una formidable base para la exportación, imposible de emular por cualquier otra empresa en la región 9 .

Las relaciones entre empresas televisivas y estado han sido fundamentales en el fortalecimiento de las primeras, no sólo en períodos recientes sino a lo largo de la historia de las industrias de medios en América Latina (Fox, 1997; Waisbord, 1995, 1998). Es imposible entender la evolución de la industria televisiva sin comprender esta articulación entre poder privado y público. Es necesario corregir la conclusión que la globalización debilita al estado en materia de medios y lo hace impotente frente a dinámicas globales. La política de medios a nivel del estado es de crucial importancia para entender la industria de televisión. Es innegable que fuerzas globales penetran y moldean la estructura doméstica de medios pero ésta es un complejo producto que no es la expresión de flujos internacionales, sino fundamentalmente el resultado de políticas que tienen a actores locales como protagonistas principales. Tanto los capitales como las empresas mediáticas operan en escala global. Esto no implica que la política nacional o el estado sean inconsecuentes o meros apéndices; por el contrario, son vitales tanto para la consolidación y la expansión de grupos domésticos y, potencialmente, como arenas para la reestructuración de la televisión o la implementación de alternativas.
Durante los noventa, las diferencias entre los países esbozadas en el pasado se acentuaron, creando una estructura de tres niveles segun diferencias en la cantidad de producción y exportación de horas de televisión: el primer grupo esta compuesto por Brasil, Mexico y, en menor medida Venezuela, el segundo por Argentina, Chile, Colombia, y Peru, y el tercero por Bolivia, Ecuador, Paraguay, Uruguay y los países de America Central y el Caribe. Es importante mencionar que, los tratados de integración económica no han tenido, hasta el momento, mayor impacto en la conformación de este esquema. Ni NAFTA ni el MERCOSUR han modificado o variado el curso de desarrollos que ya estaban en proceso y continuaron intactos después de la firma de los tratados. NAFTA no varió la posicion de los intereses domésticos en términos de propiedad y contenido en Mexico y MERCOSUR prácticamente ignoró cuestiones pertinentes a las industrias audiovisuales (ver Galperín 1999).

\section{CONCLUSIONES}

El aumento de la producción local y regional debe entenderse como resultado de factores económicos, políticos y culturales. La globalización mediática impacta a los sistemas de televisión en la región en términos diferentes.

No todas las televisiones nacionales son igualmente vulnerables al tsunami de la globalización. La globalización mediática no es unidireccional Norte-Sur sino que implica flujos cruzados, fundamentalmente, movimientos de capital y programas de mercados grandes (tanto regionales como mundiales) hacia mercados menores. En mercados grandes y medianos, las grandes empresas no solo pueden resistir el embate global sino que también se benefician al formar alianzas estratégicas con firmas internacionales que les permite fortalecer su poder a nivel local y nacional. Las políticas de libre mercado sumadas a la eliminación de barreras comerciales en varios países ofrecen condiciones favorables para la expansión de la producción doméstica. Mercados pequeños con empresas débiles para estandares regionales son mas vulnerables a los flujos de capital y programación que mercados medianos y grandes, particularmente aquellos que tienen empresas firmemente consolidadas en distintas áreas de medios. Todos los mercados son proclives a la entrada de programación extranjera (sobre todo regional) durante épocas cuando la inversión publicitaria cae abruptamente debido a descalabros financieros que hace que los canales recorten gastos y prefieran opciones baratas. En

8. El caso de Guatemala donde, según el cuadro, las producciones locales son más frecuentemente vistas que las regionales presenta una anomalía ya que la producción de drama es escasa.

9. En el caso de Televisa, el hecho que las audiencias regionales estuvieran acostumbradas al acento mexicano después de décadas de doblajes de producciones de Hollywood, eliminó una importante barrera cultural. Producciones mexicanas no son tan foráneas en la región como lo son, por ejemplo, las producciones argentinas o colombianas. Además tanto Globo como Televisa patentaron un género como la telenovela que además de tener contenidos fácilmente entendibles de país a país, es extremadamente adecuado a las necesidades de los programadores mundiales ya que es ficcion seriada, renovable anualmente y, si es exitosa, posible de ser continuada en temporadas subsequentes. 
varios países, tales decisiones han ocasionado protestas de sindicatos de actores por falta de trabajo debido a la inclinación de los canales por programas de juego, talk-shows y de variedades, pedidos de proteccionismo e incluso, irónicamente en una región donde producciones norteamericanas solían ser vistas como el peligro de extranjerización, fuertes críticas contra la presencia de programas regionales.

Asimismo, la historia reciente de la televisión latinoamericana muestra que el estado y la política doméstica tienen un papel importante en controlar flujos internacionales y en definir como compañías y mercados locales se posicionan frente a la globalización. Las afirmaciones sobre la desaparición del Estado y la política en la globalización son, como los reportes de la muerte de Mark Twain, exagerados. El Estado todavía controla recursos claves para intereses comerciales, lo cual explica porque empresas domésticas (y globales) cortejan a gobiernos para influir políticas que afectan sus planes no sólo en la industria televisiva sino de expansión en el área de telecomunicaciones donde, debio a la liberalización y desregulación, las ganancias son varias veces mayor que en los medios tradicionales.

Diferenciar los efectos de la globalización según países no implica juzgarla positivamente para la salud de las industrias televisivas de la región. Estos juicios dependen de las expectativas que se tengan sobre la televisión. Si las preocupaciones son las chances que la televisión latinoamericana tenga producción local que tenga resonancia con culturas nacionales y refleje intereses de las audiencias domésticas, las posibilidades no son tan dramáticas en algunos casos como sugieren argumentos pesimistas que ven en la globalización mediática el fin de las industrias televisivas nacionales. Mas que la globalización mediática, las sucesivas crisis financieras sumadas al subeybaja de la economía han notablemente minado las posibilidades para el afianzamiento de la producción local en la última década. Ambiciosos planes de incrementar producción basados en pronósticos optimistas sobre montos de inversiones publicitarias fueron cambiados y reducidos después que las debacles financieras golpearan las economías de la región. No obstante el aparente esplendor de la producción local, diagnósticos recientes son sombríos, describiendo una industria en crisis y que, desde el punto de vista comercial, no es redituable.

Es necesario afinar el análisis y reconocer que la situación de la televisión depende de contextos políticos y económicos que varían de país en país. Las fronteras nacionales de las economías, en efecto, se desdibujan debido al impacto de nuevas tecnologías para la distribución de programas y políticas que facilitan el movimiento de capitales y de programación. Esto no implica la extinción sino la complejización del mapa regional de la producción televisiva. En países donde las condiciones han sido históricamente desfavorables para el afianzamiento de la producción doméstica no sólo económicamente sino también debido a la falta de medidas proteccionistas, la globalización debilita mas aun las chances de una producción ligada a culturas locales. En otros países, sin embargo, la sumatoria de condiciones económicas, culturales y políticas ofrecen una suerte de vallado frente a flujos globales. En contextos de industrias cada vez mas concentradas, un manojo de empresas se constituyen como dominantes. Si bien éstas no estan particularmente interesa- das en la diversidad y el pluralismo de ideas sino en obtener réditos comerciales, su poderío sumado a la preferencia de la audiencias por verse en las pantallas se tradujo en el aumento de la producción, aun cuando capitales y programas globales cruzen las fronteras con mas facilidad que antes.

\section{BIBLIOGRAFÍA}

Álvarez, Luciano (1993): La casa sin espejos: Perspectivas de la industria audiovisual uruguaya. Montevideo: Fin de Siglo.

AthaYde, Antonio (1999): "A TV brasileira e os investidores estrangeiros”. Tela Viva, Septiembre.

Audits \& Surveys (1998): "Los medios y mercados de Latinoamérica".

Blejman, Mariano (1999): "IBOPE pasó el proceso de homologación y quedó como la única medidora autorizada”. www.pagina12.com.ar/1999/99-12/99-12-22/pag25.htm

CAPITAL (1998): "Carta de ajuste". www.capital.cl/1998/ medio28.htm

Catalán, Carlos y María Dolores Souza (1999): Calidad, identidad y televisión. CNTVNT1.CNTV.CL/CNTV

CONNOR, Michael (1997): “USA network's CEO sees fast Latin ad growth". En Reuters, 4 de Febrero.

IBOPE (1999a): Ratings. Ibope.com.co/top20.htm

IвOPE (1999b): Los diez mejores programas. www.ibopeagb.com.mx/sem $4499 . h$ tm

Fox, Elizabeth (1997): Latin American broadcasting: From tango to telenovela. Luton: John Libbey Media y University of Luton Press.

GALPERÍNN, Hernán (1999): "Cultural industries in the age of freetrade agreements”. En Canadian Journal of Communication 24, 49-77.

Hoskins, Colin and R. Mirus (1988): "Reason for the US dominance of the internartional trade in television programmes." En Media, Culture and Society 10: 499-515.

Hoskins, Colin, Stuart McFAyden, y Adam Finn (1997): Global television and film. Oxford: Oxford University Press.

KILBY, Paul (1997): "The message is media". En Latin Finance, Junio, 33.

LA REPúBLICA (1999): "Rating manda". www.larepublica.com. pe/diario/televicio.htm

Paxman, Andrew (1999a): "TV tangos with indies". En Variety, Marzo 29-Abril 4, p. 49.

Paxman, Andrew (1999b): "Latino satcasters ponder long haul" En Variety, Abril 19-25, p. 32

PAXman, Andrew y Mary SutTer (1997): "Soap cleans up for Azteca”. En Variety, Octubre 13-19.

Semana (1999): "La quiebra de la TV”. 21 de Junio, www.semana.com/users/semana/semana99/jun21/nacion3.htm

SinClair, John (1999): Latin American television: A global view. Oxford: Oxford university press.

WAISBORD, Silvio (1995): "Leviathan dreams: State and broadcasting in Latin America”. En The communication review 1 (2): 201-226

WAISBORD, Silvio (1998): "The ties that still bind: Media and national cultures in Latin America”. En Canadian journal of communication, 23: 381-401.

WATERMAN, David (1988): "World television trade: The economic effects of privatization and new technology". Telecommunications Policy, Junio, 141-51.

WILDMAN, Steve (1995): "Trade liberalization and policy for media industries: A theoretical examination of media flows". En Canadian Journal of Communication, 20: 367-88. 